\section{A notch for IGF}

The fragment of the type I insulin-like growth factor receptor (IGF-1R) for which the structure has just been determined (residues 1-462; reported by Garrett et al. Nature 394, 395-399; 1998) is incapable of binding to its ligand, insulin-like growth factor. This, initially, might seem a disappointment, raising the question: what then is the relevance of the structure? The answer is a great deal.

The fragment, which comprises about half of the ectodomain of the IGF-1R, is composed of three domains: two righthanded $\beta$-helix motifs, L1 ( $1-150$; top) and L2 (300-460; bottom); and an elongated cysteine-rich region (middle). A first glance would suggest that the large notch in the structure with the prominently placed loop (255-265) would be a likely place for ligand to bind. Such a presumption turns out to be correct.

Studies of chimeric receptors reveal that residues 1-68 of the insulin receptor (IR, which exhibits very considerable homology with IGF-1R) confer insulin binding in the context of the IGF-1R. Conversely, residues 191-290 of IGF-1R confer IGF binding in the context of IR. The authors note that a ligand bound in the notch could contact both these regions. Mapping of the residues that are important for insulin binding in the IR (and, by extension, IGF-1R given their similarity) reveal they cluster in three areas: on the underside of the L1 domain (indicated by the arrow); in the $\mathrm{N}$-terminal region of the L2 domain (upper portion of L2 in the picture), where a mutation causing Rabson-Mendenhall syndrome and severe insulin resistance is found; and within the central cys-rich region, where residues 223-274 are involved in determining ligand specificity (including the 255-265 loop). All three of these sites face into the notch, supporting the idea that this is where IGF binds.

In the structure the notch is too wide ( 30 A between L1 and L2) for the three faces to bind ligand simultaneously. The picture clearly shows that the position of the L2 domain is not constrained by interactions with the other two domains, rather it is probably crystal packing effects that dictate the overly large size of the notch. Thus, even though this fragment of the ectodomain is not sufficient to bind IGF, the notch

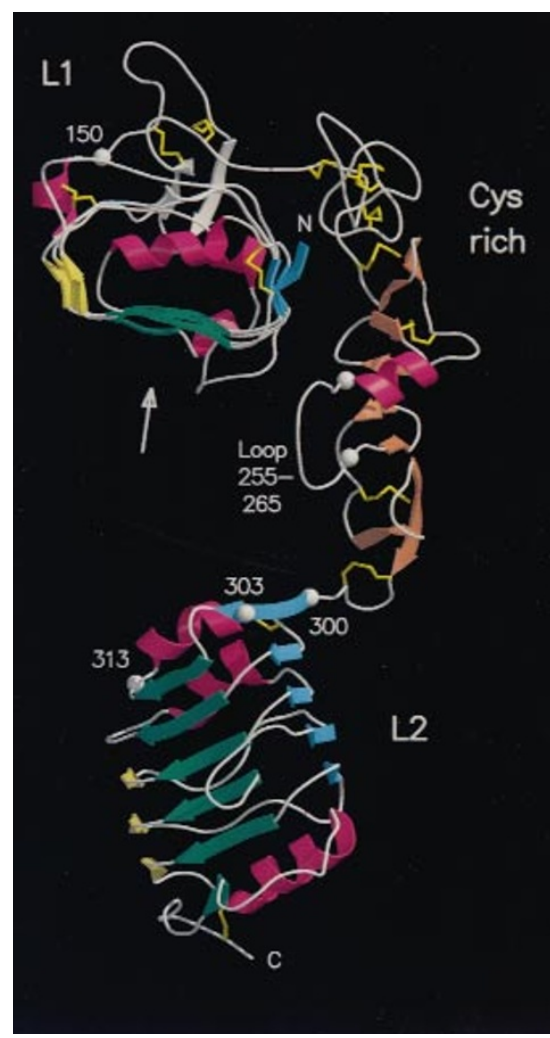

does bare the major specificity determinants necessary for the interaction with ligand. 